\title{
TENDÊNCIAS DAS PESQUISAS DE ENFERMAGEM EM SAÚDE DA MULHER NO PERÍODO DE 2001 A 2005 ${ }^{1}$ TENDENCIES IN NURSING RESEARCHES ON WOMAN'S HEALTH FROM 2001 TO 2005 TENDENCIAS DE LAS INVESTIGACIONES DE ENFERMERÍA EN SALUD DE LA MUJER EN EL PERIODO DE 2001 A 2005
}

\author{
Clándia Maria Ramos Medeiros Souto², Sarah Maria Fraxe Pessoa², Marta Maria Coelho Damasceno ${ }^{3}$, Thelma Leite Araújo
}

\author{
${ }^{1}$ Pesquisa realizada como requisito da disciplina “Análise da Pesquisa em Enfermagem” do Programa de Pós-Graduação em \\ Enfermagem da Universidade Federal do Ceará (UFC). \\ ${ }^{2}$ Enfermeira. Doutoranda do Programa de Pós-Graduação em Enfermagem da UFC. Ceará, Brasil. \\ ${ }^{3}$ Doutora em Enfermagem. Docente do Programa de Pós-Graduação em Enfermagem da UFC. Pesquisadora do Conselho \\ Nacional de Desenvolvimento Científico e Tecnológico. Ceará, Brasil.
}

PALAVRAS-CHAVE: Saúde da mulher. Enfermagem. Violência. Gravidez.
RESUMO: Objetivou-se analisar as tendências das pesquisas na área da saúde da mulher, em resumos publicados nos Anais dos Congressos Brasileiros de Enfermagem, no período de 2001 a 2005. Estudo bibliográfico cujos dados foram coletados, utilizando-se um formulário estruturado sendo a amostra constituída por 960 resumos. Os resultados foram analisados com o auxílio da literatura específica e da estatística descritiva e apontaram tendência aos estudos qualitativos, com mulheres adultas, realizados em hospitais, provenientes da região sudeste, com predomínio de autores docentes seguidos de discentes de graduação e enfermeiros assistenciais. As temáticas gestação, parto, câncer de mama e útero, aleitamento materno, doenças sexualmente transmissíveis e síndrome da imunodeficiência adquirida guardam relação com as políticas vigentes, o que reflete uma tentativa de responder às necessidades de saúde das mulheres e a perspectiva de aliar conhecimento científico à atenção humanizada. Foram observadas lacunas em áreas prioritárias como a saúde da mulher idosa, violência e gênero.
KEYWORDS: Woman's health. Nursing. Violence. Pregnancy.
ABSTRACT: The objective of this study was to analyze research tendencies in woman's health, through abstracts published in the Annals of Brazilian Nursing Conferences from 2001 to 2005. This is a bibliographic study whose data was collected by using a structured form, with the sample constituting of 960 abstracts. Results were analyzed with help of the specific literature and descriptive statistics which showed a tendency towards qualitative studies, with adult women participants, carried out in hospitals, and from southeastern Brazil, in which authorship faculty were predominant followed by undergraduate students and nursing assistants. The themes pregnancy, labor, breast and womb cancer, breast feeding, sexually transmitted diseases, and acquired immunodeficiency syndrome keep a relation with current policies, which reflects an attempt to respond to women's health needs and the perspective of joining scientific knowledge to humanized attention. The study pointed out gaps in important areas like elderly women's health, violence, and gender.
PALABRAS ClaVE: Salud de la mujer. Enfermería. Violência. Embarazo.
RESUMEN: El objetivo de este estudio fue analizar las tendencias de las investigaciones en el área de la salud de la mujer, en resúmenes publicados en los Anales de los Congresos Brasileños de Enfermería, en el período de 2001 a 2005. Es un estudio bibliográfico cuyos datos fueron recolectados a través de formulario estructurado, y la muestra constituida por 960 resúmenes. Los resultados obtenidos se analizaron con auxilio de la literatura específica y de estadística descriptiva, los cuales señalaron tendencia a los estudios cualitativos, realizados en hospitales, con mujeres adultas provenientes de la región sudeste, con predominio de autoras docentes, seguidas por las alumnas de pregrado y de enfermeros asistenciales. Las temáticas: embarazo, nacimiento, cáncer de mama y útero, lactancia materna, enfermedades sexualmente transmisibles y síndrome de inmunodeficiencia adquirida se relacionan con las políticas existentes, lo que refleja un intento de responder a las necesidades de salud de las mujeres y a la perspectiva de relacionar el conocimiento científico con la atención humanizada. La investigación reveló vacíos en áreas prioritarias tales como la salud de la mujer anciana, violencia y género.
Sarah Maria Fraxe Pessoa

Endereço: Av. Rui Barbosa, 3288

60.115-222 - Joaquim Távora, Fortaleza, CE, Brasil.

Email: sarahfraxe2005@yahoo.com.br
Artigo original: Pesquisa

Recebido em: 16 de abril de 2007

Aprovação final: 22 de outubro de 2007 


\section{INTRODUÇÃO}

As reflexões sobre as tendências na produção científica de enfermagem na área de saúde da mulher surgem, sobretudo, das inquietações sobre o modo como a profissão, enquanto prática social, vem enfocando e respondendo às questões da saúde feminina em suas pesquisas. Para o crescimento da profissão, é relevante conhecer quantitativos e qualitativos da produção científica em geral e das áreas de saber específicas, na procura de evidências não somente sobre o desenvolvimento profissional, mas, fundamentalmente, sobre os resultados e impactos do progresso científico e tecnológico na saúde da população feminina. ${ }^{1}$

As tendências da investigação científica devem ser analisadas à luz de sua correspondência com a realidade, levando em conta os aspectos político, econômico, social, cultural e histórico a que esse processo de construção do conhecimento se submete inevitavelmente. A implantação da Reforma Universitária Brasileira na década de 70 favoreceu fortes mudanças no ensino de graduação, concomitantemente à implantação da Pós-Graduação stricto sensu, que possibilitou o envolvimento mais efetivo das enfermeiras com a pesquisa e com a produção de trabalhos científicos. ${ }^{2}$ A partir de então, a produção científica da enfermagem é procedente, sobretudo, dos cursos de pós-graduação, e a criação destes permitiu um avanço significativo na avaliação crítica da prática profissional, notadamente a partir da década de 80 .

Marcos históricos como o movimento feminista, a reforma sanitária brasileira, a criação do Programa de Assistência Integral à Saúde da Mulher (PAISM); eventos internacionais como as conferências no Cairo e em Beijing e, ainda, a Convenção do Pará, são aspectos relevantes para análise das tendências de investigação nesse campo específico do saber.

Atuar no ensino, na pesquisa e na assistência na área de saúde da mulher, significa vivenciar um processo de reflexão sobre o modo como as questões de saúde da mulher vêm sendo contempladas na investigação científica, ou seja, as temáticas predominantes, os locais de produção de pesquisas, os atores das investigações e as abordagens teórico-metodológicas.

O Congresso Brasileiro de Enfermagem (CBEn) identifica-se como um meio de veiculação das pesquisas por sua significativa contribuição desde 1947, na produção e divulgação do conhecimento em diversos campos do saber na enfermagem. $\mathrm{O}$ seu acervo está disponível em Anais, um meio de socialização do conhecimento, que enfoca a saúde da mulher como uma de suas temáticas, dando-lhe significativa contribuição.

A partir da indagação sobre o modo como as pesquisas em saúde da mulher vêm sendo produzidas na enfermagem, este estudo se propôs a analisar as tendências das pesquisas na área, publicadas nos Anais dos Congressos Brasileiros de Enfermagem, no período de 2001 a 2005.

\section{MATERIAL E MÉTODOS}

Para atender a proposta de investigação, desenvolveu-se uma pesquisa bibliográfica pois é adequada para analisar publicações e identificar, entre outros aspectos, a sua freqüência, regularidade, tipos, assuntos examinados, e métodos empregados. ${ }^{3}$

A população do estudo foi constituída de resumos publicados nos Anais dos Congressos Brasileiros de Enfermagem, no período de 2001 a 2005, localizados em CD ROM (2001 a 2003) e disponíveis on-line (2004 e 2005), nos quais os sujeitos fossem mulheres e o enfoque abrangesse a saúde sexual e reprodutiva, gênero e violência, o que resultou em 1.494 resumos. Posteriormente, acrescentou-se outro critério de inclusão, ou seja, tratar-se de resumos de relatórios de pesquisa, o que significou excluir notas prévias e reflexões, perfazendo um total de 960 resumos. $\mathrm{O}$ recorte do período estudado justifica-se por assegurar a atualidade dos dados, mostrando o interesse e a tendência das investigações analisadas.

Os dados foram coletados de novembro de 2005 a fevereiro de 2006, mediante a utilização de um formulário estruturado, abrangendo questões condizentes com a proposta da pesquisa, incluindo: métodos de estudo adotados, sujeitos das pesquisas, cenário da investigação, região do país em que a investigação se realizou, área de atuação dos autores e temáticas abordadas.

Os dados foram analisados utilizando-se a estatística descritiva, além disso, considerou-se as diretrizes e ações programáticas preconizadas pelas políticas públicas vigentes na área da saúde da mulher, bem como a literatura disponível sobre as tendências das pesquisas de enfermagem.

\section{RESULTADOS E DISCUSSÃO}

Encontrou-se, na Tabela 1, uma predominância da abordagem qualitativa nas pesquisas, considerado um achado adequado à produção do conhecimento em enfermagem para subsidiar as 
ações práticas, uma vez que possibilita maior interação entre pesquisadores e pesquisados, favorecendo maior compreensão do objeto estudado. ${ }^{4}$

Tabela 1 - Distribuição dos resumos publicados sobre saúde da mulher segundo as características. Anais dos Congressos Brasileiros de Enfermagem - 2001 a 2005. ( $=960)$. Fortaleza - CE, 2006.

\begin{tabular}{|c|c|c|}
\hline Características & $\mathbf{n}$ & $\%$ \\
\hline \multicolumn{3}{|l|}{ Tipos de estudo } \\
\hline Qualitativo & 552 & 57,50 \\
\hline Quantitativo & 172 & 17,91 \\
\hline Quanti-qualitativo & 93 & 9,69 \\
\hline Não informado & 143 & 14,90 \\
\hline Total & 960 & 100 \\
\hline \multicolumn{3}{|l|}{ Sujeitos/mulheres ${ }^{*}$} \\
\hline Adulta & 325 & 31,68 \\
\hline Adolescente & 237 & 23,09 \\
\hline Idosa & 50 & 4,88 \\
\hline Não informado & 414 & 40,35 \\
\hline Total & 1026 & 100 \\
\hline \multicolumn{3}{|l|}{ Cenários* } \\
\hline Instituições hospitalares & 394 & 39,64 \\
\hline Unidade básica & 215 & 21,63 \\
\hline Arquivo/base de dados & 116 & 11,68 \\
\hline Comunidade & 68 & 6,84 \\
\hline Escola/Universidade & 43 & 4,32 \\
\hline Empresa/Instituição/ONG & 42 & 4,22 \\
\hline Domicílio & 28 & 2,82 \\
\hline Não informado & 88 & 8,85 \\
\hline Total & 994 & 100 \\
\hline \multicolumn{3}{|l|}{$\overline{\text { Regiões }^{\star *}}$} \\
\hline Sudeste & 359 & 37,78 \\
\hline Nordeste & 297 & 31,30 \\
\hline Sul & 180 & 18,94 \\
\hline Centro-oeste & 38 & 4,00 \\
\hline Norte & 21 & 2,21 \\
\hline Não informado & 55 & 5,78 \\
\hline Total & 950 & 100 \\
\hline Autores $^{* * *}$ & & 39,91 \\
\hline Docente & 620 & 32,00 \\
\hline Discente de Graduação & $\begin{array}{l}0<0 \\
303\end{array}$ & 15,65 \\
\hline Assistencial & 160 & 8,26 \\
\hline Discente de Mestrado/Doutorado & 21 & 1,09 \\
\hline Discente de Especialização & 19 & 0,99 \\
\hline $\begin{array}{l}\text { Outros profissionais } \\
\text { Não informado }\end{array}$ & 41 & 2,11 \\
\hline Total & 1937 & 100 \\
\hline $\begin{array}{l}\text { Encontrou-se em diversos estu } \\
\text { sujeito, cenário e área de atuaçã }\end{array}$ & $\begin{array}{l}\text { ais de } \\
\text { autores. }\end{array}$ & tipo de \\
\hline $\begin{array}{l}\text { Encontrou-se estudos em r } \\
\text { colaboração internacional (10). }\end{array}$ & brasil & as com \\
\hline $\begin{array}{l}* * * \text { Ausentes os dados do ano } 2005 \\
\text { por falta de informações nos Ar }\end{array}$ & atuação & autores \\
\hline
\end{tabular}

Acredita-se que a tendência à abordagem qualitativa seja um reflexo do desenvolvimento galgado pela Enfermagem como profissão desde a modernidade com Florence, voltando-se muito mais para influências filosóficas como o humanismo e a fenomenologia, e muito menos para o positivismo que foi se mostrando inadequado à busca de compreensão e explicação dos fenômenos sociais, fazendo emergir novas abordagens metodológicas nas investigações científicas.

Resultados semelhantes foram encontrados nos catálogos do Centro de Estudos e Pesquisas em Enfermagem (CEPEn), em que os pesquisadores enfermeiros vêm utilizando marcos referenciais teórico-filosóficos com predominante abordagem sócio-humanística, indicando uma riqueza de dados que podem ser utilizados para construir conhecimentos sobre a temática do cuidado, vislumbrando um olhar holístico do ser humano. ${ }^{5}$ Entende-se que a enfermagem enquanto prática social de atenção à saúde que lida diretamente com o ser humano e toda a sua complexidade - sobretudo no campo de atenção à saúde da mulher - deva ter a prática do cuidado e da investigação voltada para uma escuta sensível, solidária e acolhedora e, com isso, apresente-se com tendência para as investigações qualitativas.

Outro aspecto observado é o significativo número de resumos nos quais está ausente o método de pesquisa adotado pelos autores, além da falta de outras informações de importância metodológica. Sobre esse dado, um outro estudo aponta a dificuldade encontrada no decorrer da exploração dos resumos, os quais não ofereceram em seus conteúdos, informações completas e objetivas, de maneira a contemplar os critérios básicos que estão recomendados nas normas técnicas vigentes. ${ }^{4}$ Dificuldades semelhantes relacionadas ao conhecimento de metodologias de pesquisa foram identificadas em outros estudos a partir da segunda metade da década de 90 , do século XX. Nesses, foram constatadas carências quanto à formação específica em metodologia de pesquisa no campo do gênero, da sexualidade e da saúde reprodutiva, apesar de razoável produção de conhecimentos. ${ }^{6}$ Esses índices apontam a necessidade de aprimoramento acadêmico na formação sobre este aspecto específico.

Quanto aos sujeitos, constata-se que a maioria das pesquisas envolveu mulheres adultas seguidas de adolescentes e idosas, tendência que se manteve ao longo do período estudado. $\mathrm{O}$ predomínio de estudos em que os sujeitos são mulheres adultas se mantém desde a criação do 
Programa Materno-Infantil. ${ }^{7}$ Nos anos setenta do século passado, que priorizava ações direcionadas às mulheres no ciclo gravídico-puerperal e embora tenha sido considerado um avanço, não deu conta das demandas de atenção à saúde do grupo. Outras faixas etárias e a atenção à saúde do homem têm merecido enfoque, mas só recentemente têm sido contemplados nas políticas e nas ações de saúde. Culturalmente ainda é a mulher - no ciclo reprodutivo - a cuidadora e provedora da saúde dos filhos e da família em geral e é ela que forma vínculo com o sistema de saúde, portanto, constata-se sua presença predominante nas pesquisas.

Os estudos com adolescentes também apareceram apontando uma tendência a ampliação de ações direcionadas a esse grupo, em decorrência do número acentuado de gestações não planejadas, abortamentos, Doenças Sexualmente Transmissíveis (DST), entre outros, justificando o planejamento de ações intersetoriais nos campos da saúde, educação, ação social, trabalho, lazer, dentre outros. ${ }^{4}$

Observou-se um baixo e decrescente número de pesquisas com mulheres idosas. Os resultados evidenciaram uma contradição com as tendências de crescimento da população brasileira, que tem aumentado sua expectativa de vida fazendo com que as necessidades de cuidado e de pesquisas aumentem e constitua um peso representativo na atenção à saúde. Problemas como o câncer de mama e de colo uterino, outros relacionados com a vivência da sexualidade como a prevenção da Síndrome da Imunodeficiência Adquirida (SIDA), das DST em mulheres da terceira idade com vida sexual ativa, e todos os fenômenos vivenciados nesta faixa etária tem importância relevante no campo das pesquisas com possibilidade de estarem subsidiando o planejamento de políticas públicas e a oferta de serviços e ações direcionadas a saúde da mulher. ${ }^{8}$

Contatou-se um elevado percentual dos estudos em instituições hospitalares representadas por hospitais, maternidades e ambulatórios, com forte tendência a sua extensão para os serviços de atenção básica e para a comunidade. Pesquisa realizada na década de 80 mostrou resultados semelhantes. ${ }^{6}$ Sobre esse aspecto, é importante ressaltar a influência dos movimentos político-ideológicos ocorridos a partir da década de 80 , do século passado. A criação do PAISM em 1983, considerado um marco na história das políticas públicas para as mulheres, tinha como uma de suas proposições a expansão e consolidação da rede básica de saúde e a Reforma Sanitária Brasileira, iniciada na mesma década que desencadeou uma profunda mudança no setor saúde no país e influenciou a expansão da atenção básica. ${ }^{9}$

O movimento sanitário que culminou com a criação do Sistema Único de Saúde (SUS) em 1988 contemplava a abrangência e o acesso das mulheres aos serviços de saúde. Outro fato marcante foi a criação do Programa Saúde da Família (PSF) em 1994, compreendido como uma estratégia de organização para a atenção básica. ${ }^{10}$ Todos esses movimentos influenciaram o modo de pensar, de planejar e de atuar no setor saúde e, sobretudo, no campo da saúde da mulher.

A manutenção das investigações nas instituições hospitalares reflete em parte a herança do modelo hegemônico de assistência à saúde e da formação de recursos humanos para a área, centrada na doença e na cura e conseqüentemente na valorização desses serviços como provedores de pesquisa e de assistência. Contudo, há uma nítida tendência avaliada como positiva para ampliação dos contextos sociais em que a enfermagem atua e uma mudança paradigmática, reflexos dos movimentos sociais, sugerindo uma mudança de concepção do conceito de saúde. ${ }^{11}$

As pesquisas em enfermagem - enquanto campo de investigação científica - devem ser analisadas à luz de sua correspondência com a realidade para poder emitir critérios a respeito de seu papel social como profissão. ${ }^{9}$ Sendo assim, considerou-se que a expansão e extensão dos cenários de investigação além de evidenciarem mudanças na atenção à saúde, mostram uma ampliação de setores e serviços em que a enfermagem pode e deve atuar, validando sua importância enquanto prática social em todos os níveis - primário, secundário e terciário - de atenção à saúde da mulher.

Houve uma tendência de elevação do número de trabalhos por região coincidente com o local de realização do evento. Refletindo sobre os motivos para os índices encontrados, enfatiza-se a facilidade na participação, no envio e apresentação dos resumos pelos autores e co-autores, bem como enfoca-se o surgimento de Programa de Pós-Graduação, na região, que já poderia estar refletindo nessa mudança de perfil.

A região sudeste apresenta o maior número de resumos publicados nos eventos analisados e, em contrapartida, a região norte permaneceu em todo o 
período estudado com o menor número de resumos apresentados nos CBEn. Os achados do estudo se assemelham e são validados em outros já publicados, os quais correlacionam a expansão nacional e regional de publicações, com o elevado número de cursos/programas de mestrado e doutorado, dados que retratam a hegemonia da região sudeste. $.^{1-2,5} \mathrm{Nes}-$ sa linha reflexiva ressalta-se que a região norte do Brasil, até o presente ano, não conta com nenhum programa de pós-graduação.

Observou-se a predominância de pesquisas de autoria de docentes seguida daquelas cujos autores são estudantes de graduação e enfermeiros assistenciais.

A produção científica na enfermagem é quantitativamente incipiente em decorrência do reduzido número de profissionais titulados, considerando-se o número de enfermeiros (as) cadastradas no Conselho Federal de Enfermagem (COFEn)/Conselho Regional de Enfermagem (COREn). Porém, a pesquisa em enfermagem expandiu-se nos últimos anos, evoluindo de uma visão predominantemente tecnicista para uma análise profunda dos problemas pesquisados, sendo acrescentadas nos estudos, as áreas biológicas, humanas e sociais. Outro aspecto relevante que reforça os achados da pesquisa, devese à atual oferta de vagas nos cursos de mestrado e doutorado para capacitação de enfermeiras(os), fortalecendo os núcleos de pesquisa existentes nas instituições e, ao mesmo tempo, incentivando o discente a publicar artigos científicos a partir dos resultados de seus estudos. ${ }^{2}$

Destaca-se no estudo, a participação de outros profissionais não enfermeiros (médicos, psicólogos, assistentes sociais, administradores, nutricionistas e pedagogo) o que aponta para uma crescente formação de núcleos de pesquisas interinstitucional e interdisciplinares, fato observado a partir da década de 90 , em que começa a se organizar a produção grupal entre docentes de uma mesma instituição ou interinstitucional, de serviços ou demais profissões. ${ }^{2}$

Essas parcerias poderiam influenciar em transformações significativas na assistência e no elo docência e assistência/teoria e prática, tão discutido nos âmbitos acadêmicos e institucionais como fator co-responsável pelo desenvolvimento da profissão.
Tabela 2 - Distribuição dos resumos publicados sobre saúde da mulher segundo a temática abordada. ANAIS dos Congressos Brasileiros de Enfermagem - 2001 a 2005. Fortaleza - CE, 2006.

\begin{tabular}{lrr}
\hline Temática & $\mathbf{n}$ & $\mathbf{\%}$ \\
\hline Gestação/pré-natal & 231 & 24,07 \\
Câncer & 139 & 14,48 \\
Parto & 96 & 10,00 \\
Aleitamento materno & 94 & 9,80 \\
DST/SIDA & 88 & 9,16 \\
Complicações no ciclo gravídico puerperal & 69 & 7,18 \\
Planejamento familiar & 48 & 5,00 \\
Humanização & 41 & 4,27 \\
Climatério/menopausa & 34 & 3,54 \\
Gênero & 30 & 3,13 \\
Sistematização da assistência/do cuidado & 29 & 3,02 \\
Violência contra a mulher & 28 & 2,92 \\
Puerpério & 23 & 2,39 \\
Mortalidade materna & 10 & 1,04 \\
\hline Total & $\mathbf{9 6 0}$ & $\mathbf{1 0 0}$ \\
\hline
\end{tabular}

Os dados da Tabela 2 mostram as temáticas mais freqüentemente investigadas. Gestação/prénatal, câncer, parto, aleitamento materno, DST/ SIDA, complicações do ciclo gravídico-puerperal, humanização, e planejamento familiar surgiram na prática assistencial de enfermagem, o que reflete claramente uma tentativa de responder às questões $\mathrm{e}$ às necessidades de saúde das mulheres na perspectiva de aliar o conhecimento científico a assistência em direção a um cuidado mais humanizado. $O$ mesmo resultado foi encontrado na análise de 301 resumos de teses de doutorado contidos nos catálogos do CEPEn, no período de 2001 a 2003. ${ }^{5}$

As temáticas gestação e pré-natal, parto e puerpério, aliadas à humanização congregaram o maior número de trabalhos, evidenciando uma prática profissional e de pesquisa voltadas para a atenção à saúde da mulher. Além disso, refletem a ênfase dada nas políticas adotadas pelo Ministério da Saúde (MS) que incluiu, desde 1998, a realização do parto normal sem distócia por enfermeiro obstetra, tendo como finalidade precípua reconhecer a assistência prestada pelos enfermeiros no contexto da humanização do parto, ${ }^{4}$ e o fortalecimento dessas políticas e de outras ações voltadas à saúde da mulher a partir desse ano. 
O Programa de Humanização no Pré-Natal, Parto e Nascimento, instituído a partir do ano 2000, representa uma das mais importantes estratégias para assegurar o acesso aos serviços de saúde, a cobertura e qualidade de atenção à mulher e ao recém nascido, além de propor a garantia da atitude ética e solidária dos profissionais e o acolhimento nos serviços.

Apesar dos avanços conquistados, problemas com a qualidade e sistematização do atendimento na gestação e parto estão presentes no cotidiano dessas mulheres, uma vez que se constata, por exemplo, que o aumento da cobertura de pré-natal, observada entre os anos de 1995 e 1996 em Fortaleza, não favoreceu a redução da mortalidade materna, suscitando dúvidas quanto à qualidade da assistência pré-natal. ${ }^{4}$ Em conseqüência disso, os altos índices apontam para a necessidade do desenvolvimento de pesquisas sobre o tema para redução do problema.

A gravidez na adolescência, item da temática gestação, destacou-se nas investigações como um dos mais importantes, sobretudo, pelas conseqüências provocadas na vida dessas mulheres, quais sejam, o abandono dos estudos e as profundas alterações do projeto de vida somados à exposição às DST/V'́rus da Imunodeficiência Humana (VIH), o que se confirma pela expansão da SIDA entre os jovens. ${ }^{12}$ A importância das investigações nessa área reside no fato de que existe o grande desafio de assegurar o acesso aos serviços de saúde a essas adolescentes, com atendimento integral, garantia de privacidade, confidencialidade e apoio sem emitir juízo de valor. Nesse sentido, as pesquisas com esse grupo da população revestem-se de importância.

Outra questão é a do aleitamento materno, área que a enfermagem tem colaborado, efetivamente, no enfrentamento de suas dificuldades. Sobre esse aspecto, os resumos voltaram a enfocar o ato de amamentar ao trazerem à luz de suas fundamentações, as experiências das mulheres na prática da amamentação, considerando aspectos tanto maternos como os dos recém-nascidos. Este fato representa importante contribuição e um avanço de concepção, na medida em que as pesquisas quase sempre tem privilegiado o favorecimento do recém-nascido em detrimento de ignorar a figura materna.

Outro tema investigado é o planejamento familiar no qual os problemas como a falta de acesso às informações, de atendimento sistematizado, de acesso aos insumos e aos serviços entre outros que se colocam para que o direito de ter ou não filhos seja conquistado, ainda existem e derivam gestações não planejadas, abortamentos provocados, infecções, hemorragias, cesarianas, laqueaduras tubárias comuns entre as mulheres e possivelmente evitáveis. Todavia, aliados a essa situação, ocorreram avanços significativos no campo da saúde sexual e reprodutiva. ${ }^{13}$

Esses avanços resultaram de movimentos internacionais de busca dos direitos das mulheres, que se consolidaram em grandes eventos como a Conferência do Cairo sobre população e desenvolvimento - em 1994 - e a IV Conferência Mundial sobre a Mulher, em Beijing - em 1995 - consideradas marcos fundamentais à elaboração das políticas existentes. ${ }^{14} \mathrm{O}$ desafio é transformar essas políticas em ações concretas de planejamento familiar. As DST/SIDA foram, entre todas as temáticas, as que mais se destacaram, não por acaso, pois fatores biológicos, socioculturais e assistenciais, além do efeito combinado desses, fazem com que a mulher seja mais vulnerável do que o homem quanto a possibilidade de contrair DST e sofrer seus efeitos secundários, biológicos e sociais. ${ }^{12}$

Em virtude da feminização da SIDA e das DST serem os principais fatores facilitadores da transmissão sexual do VIH e da transmissão vertical, a associação do VIH e gestação é considerada uma condição de risco. No Brasil, mais de $90 \%$ dos casos de SIDA em crianças estão relacionados à transmissão vertical, $15 \%$ a $30 \%$ das crianças que nascem de mães soropositivas, adquirem o vírus na gestação, no parto ou na amamentação. ${ }^{15}$ Além dos riscos de infecção no ciclo gravídico-puerperal, as mulheres com antecedentes ou portadoras de DST apresentam maior predisposição para câncer cérvico-uterino e para outros fatores que aumentam esse risco, como a infecção pelo Vírus do Papiloma Humano (VPH). ${ }^{12}$

O problema do câncer feminino foi tema nas investigações realizadas e a sua importância é atribuí$\mathrm{da}$ ao fato de ser a segunda maior causa de morte. Os resumos apontaram estudos nas áreas de prevenção, detecção precoce e tratamento, previstas desde a criação do PAISM. Em torno de $80 \%$ dos tumores de mama são detectados pela própria mulher com o auto-exame. $\mathrm{O}$ incentivo a sua realização justifica-se porque o conhecimento do próprio corpo aumenta as possibilidades de detecção precoce. A citologia cérvico-vaginal é um teste efetivo e de baixo custo para o rastreio do câncer e de seus precursores. $\mathrm{Na}$ maioria das vezes, é prática das enfermeiras na atenção básica e, dessa forma, essas profissionais precisam aprimorar suas pesquisas em busca de tecnologias apropriadas com vistas à redução da mortalidade por 
esses tipos de câncer, que vêm crescendo nos últimos 20 anos, e das repercussões físicas, psíquicas e sociais das mulheres acometidas pelo problema. ${ }^{16}$

$O$ aumento da expectativa de vida trouxe um incremento do número de mulheres no climatério, etapa da vida feminina que se inicia por volta dos 40 anos e se estende até os 65 anos de idade 7 . No estudo, o climatério foi o principal foco de pesquisa entre mulheres acima dos 45 anos e, sobretudo, o modo como esse período é vivenciado. Apesar da importância da temática, observou-se uma lacuna com relação à investigação de outros aspectos importantes para a saúde da mulher após sessenta anos de idade.

As pesquisas desenvolvidas com mulheres no climatério tiveram importância significativa, pois esse é considerado um tema relativamente novo. As políticas públicas de saúde voltadas a esse grupo da população ainda são incipientes e com poucos avanços. Além disso, quantitativamente, os índices de investigações realizadas não foram expressivos e os resultados apontaram a necessidade de incremento de pesquisas nessa área de atenção.

Gênero e violência foram consideradas temáticas emergentes. Definido como uma construção cultural dos atributos da masculinidade e da feminilidade, a socialização de gênero delimita o poder entre os sexos, produzindo desigualdade entre mulheres e homens. Nesse contexto, distinguem-se os papéis do homem e da mulher na família, na divisão do trabalho, na oferta dos bens e serviços e até na instituição e aplicação de normas legais, atribuindo-se aos homens mais poderes que as mulheres, o que justifica o autoritarismo masculino, interpretando-se a violência contra a mulher como um ato natural. ${ }^{13-14}$ No Brasil, a violência atinge uma entre cada cinco mulheres; grande parte é de natureza doméstica e em geral é praticada por homens com laços de intimidade com as vítimas. ${ }^{15}$ Os crescentes índices de notificação da violência através do registro nas delegacias especializadas e as políticas públicas de saúde e de segurança têm considerado a violência de gênero como uma questão prioritária. Dada a sua importância, recomenda-se que sejam incentivadas as investigações na área em busca de estratégias de enfrentamento e de reconhecimento desse tipo de violência pelas mulheres, pelos serviços e profissionais de saúde.

Analisadas à luz do modelo de assistência proposto pelo PAISM, adotado como diretriz nacional pelo MS a partir de $1985,{ }^{15}$ e que propôs ações globalmente dirigidas ao atendimento de todas as necessidades de saúde das mulheres, ${ }^{7}$ verifica-se a partir dos resultados do estudo, uma tendência à investigação de temas que guardam uma forte relação com as políticas públicas e com os problemas de saúde da mulher. Além disso, a temática "sentimentos relacionados à maternidade" aponta para a tentativa de apoiar as mulheres em busca de modos de enfrentar as necessidades emocionais e existenciais, advindas desse acontecimento, o que reflete também a busca de um modelo de assistência na perspectiva da integralidade.

Por fim, vale ainda destacar que a produção científica de enfermagem em saúde da mulher, no período estudado, representou $11,94 \%$ da produção total inserida nos CBEns investigados.

\section{CONSIDERAÇÕES FINAIS}

No delineamento das pesquisas, o estudo apontou o método qualitativo como o mais utilizado. Os significados e as experiências vividas por essas mulheres têm sido o enfoque prevalente com atenção primordial na mulher adulta e jovem. As instituições hospitalares e os serviços especializados foram os cenários mais investigados com uma leve tendência de avanços em estudos envolvendo o ambiente hospitalar como primeira fonte de investigação, chegando aos domicílios, através do PSF e das Unidades Básicas de Saúde.

A região sudeste do país prevaleceu em número de resumos publicados nos Anais, necessitando outras investigações a fim de relacionar esses dados ao número de cursos e programas de pós-graduação, fato já apontado em outras pesquisas. Os docentes e discentes de graduação foram os autores com maior prevalência, seguidos dos enfermeiros assistenciais. Foram encontradas categorias diversas de profissionais pesquisando com os enfermeiros em prol do incremento da produção científica de enfermagem. Os resultados mostraram que as temáticas mais frequentemente abordadas foram gestação e pré-natal, câncer, parto, aleitamento materno, DST/SIDA, complicações do ciclo gravídico-puerperal, humanização e planejamento familiar, o que reflete, claramente, uma tentativa de responder às questões e às necessidades de saúde das mulheres e, além disso, reflete a possibilidade de aliar o conhecimento científico à assistência em direção a um cuidado mais humanizado.

Devido à importância e à magnitude da área estudada no cenário da saúde, há uma expectativa 
de significativa participação dos enfermeiros no desenvolvimento de pesquisas que contemplem a saúde sexual e reprodutiva da mulher, enfocando as questões de gênero e violência. Apesar dos estudos já desenvolvidos, há a necessidade de incremento em pesquisas nessas temáticas. A prática da violência contra a mulher, em todas as idades, incluindo a violência doméstica e sexual, tem sido debatida não somente no campo da saúde, mas também em outras áreas como a educação, direito, trabalho e assistência social, com vistas à prevenção, ao enfrentamento da violência e ao tratamento de danos e patologias conseqüentes.

Consideram-se escassas, ainda, as investigações que enfocaram o climatério, o que representa uma lacuna significativa em virtude do aumento da expectativa de vida das mulheres e dos problemas que costumam surgir nesse período.

Apesar dos avanços obtidos na atenção à saúde das mulheres brasileiras, em grande parte como resultado da força de movimentos sociais, sobretudo o feminista, aliados às pesquisas e às políticas públicas no campo da saúde, é prioritário dar continuidade à realização de investigações nas temáticas já contempladas e, ao mesmo tempo, investir no desenvolvimento de pesquisas, direcionadas àquelas até então pouco estudadas. Analisando atentamente a saúde sob o viés $\mathrm{da}$ integralidade e da intersetorialidade, os estudos relativos à saúde da mulher devem estar também articulados a outros como os do trabalho e renda, moradia, educação, política, etc., capazes de conduzir essa mulher a um contexto social que reflita possibilidades de melhoria de qualidade de vida e de saúde.

\section{REFERÊNCIAS}

1 Tyrrel MAR, Cabral IE. A produção científica de enfermagem em saúde da mulher e da criança: panorama brasileiro. Rev. Enferm. UERJ 2005 JanAbr; 13 (1): 103-11.

2 Moura MAV, Spíndola T, Ferrer GH, Siqueira PRA, Chamilc RA. Tendências da produção científica em enfermagem na área de saúde da mulher. Esc. Anna Nery: rev. enferm. 2001 Dez; 5 (3): 335-46.

3 Leopardi MT. Metodologia da pesquisa na saúde. Santa Maria (RS): Palloti; 2001.

4 Moura ERF, Franco ES, Fraga MNO, Damasceno MMC. Produção científica em saúde da mulher na pós-graduação da Universidade Federal do Ceará, Brasil 1993-2002. Ciência Enferm. 2005 Dic; XI (2): 59-70.

5 Erdmann AL, Leite JL, Mendes IAC, Trevizan MA, Dantas CC. Análisis de investigaciones brasileñas enfocadas en el cuidado de enfermería, años 2001-2003. Ciência Enferm. 2005 Dic; XI (2): 35-46.

6 Barbosa RM, Aquino EML, Heilbor ML, Berquió E, organizadores. Interfaces: gênero, sexualidade e saúde reprodutiva. Campinas (SP): Ed. UNICAMP; 2002.

7 Ministério da Saúde (BR). Assistência integral a saúde da mulher: bases de ação programática. Brasília (DF): MS; 1984.

8 Ministério da Saúde (BR). Assistência ao climatério. Brasília (DF): MS; 1994.

9 Costa AM. Programa de Assistência Integral a Saúde da Mulher - PAISM. In: Rede Feminista de Saúde. A presença da mulher no controle social das políticas de Saúde: anais da capacitação de multiplicadores em controle social das políticas de saúde. Belo Horizonte (MG): Mazza Ed.; 2003. 82-101.

10 Antunes MJ. Saúde: direito de todos e dever do estado? In: Rede Feminista Saúde. A presença da mulher no controle social das políticas de saúde: anais da capacitação de multiplicadores em controle social das políticas de saúde. Belo Horizonte (MG): Mazza Ed.; 2003. p.102-22.

11 Huerta EDPN, Madeira LM, Miranda MM, Merighi MAB, Ho SFS, Garcia TR. A pesquisa em enfermagem materno-infantil. Rev. Esc. Enferm. USP 1983 Dez; 17 (3): 213-27.

12 Ministério da Saúde (BR). Doenças sexualmente transmissíveis. Brasília (DF): MS; 2002.

13 Ministério da Saúde (BR). Planejamento familiar: manual para o gestor. Brasília (DF): MS; 2002.

14 Ministério da Saúde (BR). Secretaria Especial de Políticas para as Mulheres. In: Anais da 1a Conferência Nacional de Política para as Mulheres, 2004 Jul 15-17; Brasília, Brasil. Brasília (DF): MS; 2004.

15 Monteiro CFS, Souza IEO. Vivência da violência conjugal: fatos do cotidiano. Texto Contexto Enferm. 2007 Jan-Mar; 16 (1): 26-31.

16 Ministério da Saúde (BR). Programa Nacional de Controle do Câncer do Colo de Útero e de Mama. Brasília (DF): MS; 2000.

17 Ministério da Saúde (BR). Violência intrafamiliar: orientação para a prática em gênero. Brasília (DF): MS; 2001. 